\title{
Study of thermal effects in two-component non-isothermal liquid chromatography considering thermally insulated columns
}

\author{
Jamil Ur Rehman, ${ }^{\dagger}$ Adeel Muneer, ${ }^{\dagger}$ Javeria N. Abbasi, ${ }^{\dagger}$ Shamsul Qamar, $,{ }^{*},+,{ }^{\dagger}$ and \\ Andreas Seidel-Morgenstern \\ $\dagger$ Department of Mathematics, COMSATS University Islamabad, Pakistan \\ ‡*Max Planck Institute for Dynamics of Complex Technical Systems, Magdeburg, Germany \\ E-mail: *qamar@mpi-magdeburg.mpg.de
}

\begin{abstract}
A two-component non-isothermal model of linear chromatography is formulated to study heat effects and interference between components in thermally insulated columns. The Laplace transform and decoupling techniques are jointly applied to solve the model equations analytically. The first and second analytical temporal moments are derived which simply vectorized the moments of a single-solute nonisothermal model. To confirm ranges of applicability of the derived analytical solutions, a high resolution finite volume method is additionally applied to solve the nonlinear non-isothermal model equations numerically. Various test problems are considered. The influence of heat transfer on the retention times and shape of the profiles are studied, as both the thermodynamics and kinetics of adsorption processes
\end{abstract}


are functions of temperature. The enthalpy of adsorption essentially influences the thermal behavior of the column. It was found that the ratio of specific heat times density of solid and liquid phases has a significant influence on the magnitude and speed of the thermal waves. The results obtained could be helpful to understand and optimize competitive adsorption chromatography.

\section{Introduction}

The chromatography has gained an enormous popularity as a separation and purification technique in the group of chemists pursuing pure and applied research, such as chemistry, biochemistry, as well as clinical and environmental sciences. This popularity is due to its capability to separate components of complex mixtures rapidly, completely, and inexpensively, even though separations of these mixtures are desired at laboratory and industrial scales. In this technique, the separation of components relies on their distinct adsorptivities to a particular adsorbent packed inside the chromatographic column. The batch liquid chromatography is the most simplest technique that involves a single cylindrical column fed periodically with the pulses of mixtures. While migrating along the column, the strongly adsorptive component stays longer inside the column as compared to the weaker adsorptive component and, hence, leaves the column afterwards.

The quantification of chromatography is complicated. When the sample is concentrated or its volume is large and the mass transfer kinetics, such as adsorption-desorption or association-dissociation with the solid phase, are rate limiting, a junk of complex problems

have to be handled simultaneously and it is difficult to find out which of them control the response of a column to a change in the composition of the mobile phase.

Multi-component models are extensions of the single-component models and the coupling of mass balances are mainly responsible for further complexity in the problem. The systems 
remain linear and uncoupled only for low concentrations. The interference due to equilibrium isotherms has been investigated by numerous authors. ${ }^{1-4}$ Also, the interference due to both isotherms and diffusion has been studied. ${ }^{5}$

Since adsorption is an exothermic process, the accompanying heat transfer can be very important in certain situations. Typically, the adsorption process has been assumed isothermal in liquid chromatography. ${ }^{6-8}$ There are only a few research articles available in the literature which deal with the non-isothermal conditions. ${ }^{9-11}$ When an eluent passes through a chromatographic column, viscous heat is generated due to friction that increases temperature to a significant degrees along the column. ${ }^{12}$ Significant effects can be due to changes in solvent viscosity or losses of column efficiency caused by radial temperature gradients. Carslaw and Jaeger ${ }^{13}$ have investigated conduction of heat in solids by deriving explicit solutions of many problems through the Laplace transformation method. Thermal effects have mostly been considered for gas chromatography ${ }^{14-17}$ and only a few contributions are devoted to the study of non-isothermal liquid chromatography. ${ }^{9-11,18-25}$ Some of the authors have made studies based on temporal moment analysis. The effects of pressure on liquid chromatography have also been discussed. ${ }^{26}$ That study showed that the effect of temperature on the constant of equilibrium must be examined under constant inlet pressure rather than at a constant flow rate to reduce the coupling effect of pressure and temperature via the temperature dependency of the viscosity.

The analytical solutions of linear models can be derived by means of Laplace transformation. ${ }^{25,27-31}$ Such solutions are only possible, when the equilibrium isotherm is linear. Although, the enhanced computing power of digital computers and the availability of accurate numerical solution techniques have reduced the significance of analytical solutions up to certain level, they still advantageously provide greater insight to the underlying physical process and can be routinely used as a design tool. Such solutions are helpful to analyze and understand the process without doing expensive and time-consuming experiments. These 
analytical solutions are helpful tools for estimating free parameters appearing in the model equations. Finally, the analytical solutions can also be used to validate the performance of newly developed numerical algorithms for more complex scenarios.

The moment generating property of the solutions in the Laplace domain can be used to obtain analytical expressions for temporal moments. Since long time, it has been found relevant and important to match analytically and experimentally determined moments for estimating model parameters. Several contributions can be found in the literature on moment analysis. ${ }^{3,8,28,29,32-41}$ The chromatography community already knows that the first two moments can be used to evaluate heights equivalent to theoretical plate (HETP) as a function of flow rate which measures the column efficiency. ${ }^{8}$ Recently, we have done a systematic study of moments of temperature responses for the single-solute elution. ${ }^{25}$ This research work extends and generalizes our recent work on the analysis of a single-solute model of non-isothermal chromatography. ${ }^{25}$ Here, we analyze a two-component EDM of non-isothermal liquid chromatography. The analytical solutions are determined by applying the Laplace transformation and eigen-decomposition technique jointly. Also, the expressions of first and second temporal moments are deduced from the Laplace-transformed solutions. Such moments are merely vectorizing those, obtained for a single-solute nonisothermal model of our previous article. ${ }^{25}$ The numerical Laplace inversion is utilized to retrieve solutions in the time domain, as analytical Laplace inversion is not feasible in this case. ${ }^{42} \mathrm{~A}$ detailed discussion about the influence of temperature on the process, inside the column is presented. The concentration and temperature profiles are presented and key parameters that influence the gradients of temperature are analyzed. Finally, a high resolution finite volume scheme (HR-FVS) is extended to solve the nonlinear non-isothermal model of liquid chromatography numerically. Both analytical and numerical results are compared to find the ranges of parameters for which our linear assumptions are held. ${ }^{24,43}$ Several practically applicable test problems are considered. 
The contents of this paper are arranged as follows. In Section 2, a two-component nonisothermal EDM is presented. The analytical solutions of the model are determined in Section 3. In Section 4, the analytical temporal moments are deduced from the Laplace domain solutions. While, in Section 5, various test problems are presented. Lastly, Section 6 gives conclusions.

\section{The mathematical model}

Assume that a two-component mixture flows along with an inert carrier through a chromatographic column homogeneously packed with spherical particles of adsorbent. The column is initially equilibrated and changes in the concentration along the radial coordinate of the column are neglected. Further, the fluid is assumed incompressible and interaction between the carrier (solvent) and stationary (solid) phases are not considered. ${ }^{8}$ The adsorption equilibrium relationships are linearized. Generally such relationships are nonlinear, however, the considered linearization is practical for small changes in the sample and can provide simple characteristic results. Here, we assume that the temperature has no effect on the physical properties like density, heat capacity, viscosity, and transport coefficients like axial heat conductivity and axial dispersion. Further, the flow rate is assumed to be independent of axial dispersion and axial heat conductivity coefficients. Because of the considered linearization of isotherm described below, the whole model is intended to be sufficiently accurate only in a limited temperature range. Thus, the physical properties are considered not to depend on temperature.

In the case of equilibrium dispersive model (EDM), equations for the mass balances in the mobile phase are given as

$$
\frac{\partial c_{i}}{\partial t}+u \frac{\partial c_{i}}{\partial z}=D_{z} \frac{\partial^{2} c_{i}}{\partial z^{2}}-F \frac{\partial q_{i}^{*}}{\partial t}, \quad i=1,2
$$


Neglecting the effects of friction and mixing, the balance law for energy in a differential volume element of a thermally insulated column is given as

$$
\left(\rho^{L} c_{p}^{L}+F \rho^{S} c_{p}^{S}\right) \frac{\partial T}{\partial t}+u \rho^{L} c_{p}^{L} \frac{\partial T}{\partial z}=\lambda_{z} \frac{\partial^{2} T}{\partial z^{2}}+\sum_{j=1}^{2} F\left(-\Delta H_{A, j}\right) \frac{\partial q_{j}^{*}}{\partial t}
$$

In Eqs. (1) and (2), $c_{i}$ and $q_{i}^{*}$ represent the $i$ th component concentrations in the liquid and solid phases, respectively. Moreover, $T$ stands for bulk temperature, $F=(1-\epsilon) / \epsilon$ shows phase ratio in terms of the total porosity $\epsilon, u$ exhibits interstitial velocity, $D_{z}$ denotes the coefficient of axial dispersion which is assumed same for both components, $\lambda_{z}$ is conductivity coefficient, $\Delta H_{A, j}$ is for enthalpy of adsorption, $c_{p}$ stands for heat capacity, and $\rho$ represents density per unit volume. The superscript $L$ is used for liquid phase, the superscript $S$ is for solid phase, whereas, $t$ and $x$ denote the time and axial coordinates. The temperature varies the amount of solute adsorbed on the solid surface that can be expressed through a van't Hoff type relation considering the enthalpy of adsorption. Therefore, the nonlinear phase equilibrium relation of the competitive Langmuir type is expressed as

$$
q_{i}^{*}\left(c_{i}, T\right)=\frac{a_{i}^{\mathrm{ref}} c_{i} e^{\left(\frac{-\Delta H_{A, i}}{R_{g}}\left(\frac{1}{T}-\frac{1}{T_{\mathrm{ref}}}\right)\right)}}{1+\sum_{j=1}^{2} b_{j}^{\mathrm{ref}} c_{j} e^{\left(\frac{-\Delta H_{A, j}}{R_{g}}\left(\frac{1}{T}-\frac{1}{T_{\mathrm{ref}}}\right)\right)}}, \quad i=1,2
$$

Here, $a_{i}^{\text {ref }}$ denotes the reference equilibrium (Henry's) constant for $i$ th component at the reference temperature $T_{\text {ref }}, R_{g}$ represents the general gas constant, and $b_{j}^{\text {ref }}$ is the coefficient of nonlinearity for $j$ th component at the reference temperature.

In order to write the mass and energy balances in a unified manner, which in turn facilitates 
our analysis, the following new variable transformations are introduced:

$$
c_{3}=T-T_{\text {ref }}, \quad q_{3}^{*}=\frac{\rho^{S} c_{p}^{S}}{\rho^{L} c_{p}^{L}}\left(T-T_{\text {ref }}\right)+\frac{1}{\rho^{L} c_{p}^{L}} \sum_{j=1}^{2} \Delta H_{A, j} q_{j}^{*}
$$

Moreover, the following dimensionless quantities are introduced to reduce the total number of variables:

$$
\begin{aligned}
& x=\frac{z}{L}, \quad \tau=\frac{u t}{L}, \quad P e_{c}=\frac{L u}{D_{z}}, \quad P e_{T}=\frac{L u \rho^{L} c_{p}^{L}}{\lambda_{z}} \\
& P e_{k}=\left\{\begin{array}{llc}
P e_{c}, & \text { if } & k=1,2, \\
P e_{T}, & \text { if } & k=3 .
\end{array}\right.
\end{aligned}
$$

Here, $L$ denotes length of the column, $P e_{c}$ and $P e_{T}$ are the respective Peclet numbers for the concentration and temperature. On using Eqs. (4), (5) and (6) in Eqs. (1) and (2), we get

$$
\frac{\partial c_{k}}{\partial \tau}+\frac{\partial c_{k}}{\partial x}=\frac{1}{P e_{k}} \frac{\partial^{2} c_{k}}{\partial x^{2}}-F \frac{\partial q_{k}^{*}}{\partial \tau}, \quad k=1,2,3
$$

Assuming small variations in the concentration and temperature, the isotherm function in Eq. (3) can be easily linearized by taking its Taylor's expansion up to first order. It is given as

$$
q_{i}^{*}\left(c_{1}, c_{2}, T\right) \approx q_{i}^{*}\left(c_{1, \mathrm{ref}}, c_{2, \mathrm{ref}}, T_{\mathrm{ref}}\right)+\left.\sum_{j=1}^{2}\left(c_{j}-c_{j, \mathrm{ref}}\right) \frac{\partial q_{i}^{*}}{\partial c_{j}}\right|_{\left(c_{1, \mathrm{ref}}, c_{2, \mathrm{ref}}, T_{\mathrm{ref}}\right)}+\left.c_{3} \frac{\partial q_{i}^{*}}{\partial T}\right|_{\left(c_{1, \mathrm{ref}}, c_{2, \mathrm{ref}}, T_{\mathrm{ref}}\right)},
$$

where $i=1,2$. After some manipulations in Eqs. (4) and (8), we obtain the following 
linear system of equations

$$
q_{k}^{*}=A_{0, k}+\sum_{l=1}^{3} A_{k l} c_{l}, \quad k=1,2,3
$$

The coefficient used in Eq. (9) can be expressed in tensor forms as

$$
A_{0, k}= \begin{cases}\frac{a_{k}^{\mathrm{ref}} c_{k, \mathrm{ref}}}{G} \sum_{j=1}^{2} b_{j}^{\mathrm{ref}} c_{j, \mathrm{ref}}, & \text { if } \quad k=1,2, \\ \frac{1}{\rho^{L} c_{p}^{L}} \sum_{j=1}^{2} A_{0, j} \Delta H_{A, j}, & \text { if } \quad k=3,\end{cases}
$$

where $G=\left(1+\sum_{j=1}^{2} b_{j}^{\text {ref }} c_{j, \text { ref }}\right)^{2}$. Moreover, for $i=1,2$

$$
\begin{aligned}
& A_{i i}=\frac{a_{i}^{\mathrm{ref}}}{G}\left(1+\sum_{\substack{j=1 \\
j \neq i}}^{2} b_{j}^{\mathrm{ref}} c_{j, \mathrm{ref}}\right) . \\
& A_{i j}=\frac{-a_{i}^{\mathrm{ref}} b_{j}^{\mathrm{ref}} c_{i, \mathrm{ref}}}{G}, \quad i \neq j . \\
& A_{i 3}=\frac{a_{i}^{\mathrm{ref}} c_{i, \mathrm{ref}} \Delta H_{A, i}}{R_{g} T_{\mathrm{ref}}^{2} G}-\frac{1}{R_{g} T_{\mathrm{ref}}^{2}} \sum_{\substack{j=1 \\
j \neq i}}^{2} A_{i j} c_{j, \mathrm{ref}}\left(\Delta H_{A, i}-\Delta H_{A, j}\right) . \\
& A_{3 i}=\frac{1}{\rho^{L} c_{p}^{L}} \sum_{j=1}^{2} A_{j i} \Delta H_{A, j}, \quad A_{33}=\frac{\rho^{S} c_{p}^{S}}{\rho^{L} c_{p}^{L}}+\frac{1}{\rho^{L} c_{p}^{L}} \sum_{j=1}^{2} A_{j 3} \Delta H_{A, j} .
\end{aligned}
$$

After introducing the values of $q_{k}^{*}(k=1,2,3)$ given by Eq. (9) in Eq. (7), we get the following system of equations:

$$
\frac{1}{P e_{k}} \frac{\partial^{2} c_{k}}{\partial x^{2}}-\frac{\partial c_{k}}{\partial x}=\sum_{l=1}^{3} \alpha_{k l} \frac{\partial c_{l}}{\partial \tau}, \quad k=1,2,3
$$

where

$$
\alpha_{k l}=\delta_{k l}+F A_{k l}, \quad k, l=1,2,3
$$


Here, $\delta_{k l}$ denotes the Kronecker delta function. The initial conditions for a column that is initially equilibrated are defined as

$$
c_{k}(x, 0)=c_{k, \text { init }}, \quad k=1,2,3 .
$$

Note that, $c_{3, \text { init }}=T_{\text {init }}-T_{\text {ref }}$. Here, $c_{i, \text { init }}(i=1,2)$ along with $T_{\text {init }}$ stands for initial concentrations and temperature within column. However, for a regenerated (cleaned) column the initial concentrations are zero, i.e. $c_{i, \text { init }}=0(i=1,2)$. In addition, the Dirichlet boundary conditions (BCs) are applied at both ends of the column:

$$
c_{k}(0, \tau)=\left\{\begin{array}{lc}
c_{k, \text { inj }}, & \text { if } 0 \leq \tau \leq \tau_{\text {inj }} \\
0, & \tau>\tau_{\text {inj }}
\end{array} \quad, \quad \frac{\partial c_{k}}{\partial x}(\infty, \tau)=0, \quad k=1,2,3 .\right.
$$

Note that

$$
c_{3, \text { inj }}=\left(T_{\text {inj }}-T_{\text {ref }}\right), \quad \tau_{\text {inj }}=\frac{u t_{\text {inj }}}{L} .
$$

Here, $t_{\text {inj }}$ stands for injection time, $c_{i \text {,inj }}$ reveals the $i$ th component concentration in the injected pulse, and $T_{\mathrm{inj}}$ represents the injected sample temperature. Generally, the values of mass and energy Peclet numbers are larger in liquid chromatography, i.e. the axial dispersion and heat conductivity coefficients are small. Therefore, the considered Dirichlet BCs are well applicable. 


\section{Derivation of analytical solutions}

The Laplace transformation can be applied to solve the suggested linearized non-isothermal EDM analytically as defined below

$$
\bar{w}(x, s)=\int_{0}^{\infty} e^{-s \tau} w(x, \tau) d \tau, \quad \tau \geq 0
$$

where $w \in\left\{c_{1}, c_{2}, c_{3}\right\}$. By applying the above Laplace transformation on Eq. (15) and utilizing the initial conditions of Eq. (17), we obtain

$$
\frac{1}{P e_{k}} \frac{\partial^{2} \overline{c_{k}}}{\partial x^{2}}-\frac{\partial \overline{c_{k}}}{\partial x}=\sum_{l=1}^{3} \alpha_{k l}\left(s \bar{c}_{l}-c_{l, \text { init }}\right), \quad k=1,2,3 .
$$

Eq. (21) contains a system of coupled equations. Therefore, our first aim is to decouple this system by applying the eigen-decomposition approach. The coefficient matrix $D$ on the right hand side of Eq. (21) is given as

$$
D=\left(\begin{array}{ccc}
\alpha_{11} & \alpha_{12} & \alpha_{13} \\
\alpha_{21} & \alpha_{22} & \alpha_{23} \\
\alpha_{31} & \alpha_{32} & \alpha_{33}
\end{array}\right)
$$

The above matrix will be diagonalizable if all the three eigenvalues are real and distinct. We get the following cubic equation:

$$
\lambda^{3}+b \lambda^{2}+c \lambda+d=0
$$

where

$$
b=-\sum_{k=1}^{3} \alpha_{k k}, \quad c=\frac{1}{2} \sum_{k=1}^{3} \sum_{\substack{l=1 \\ l \neq k}}^{3}\left(\alpha_{k k} \alpha_{l l}-\alpha_{k l} \alpha_{l k}\right), \quad d=-\operatorname{det} D
$$


Let us define

$$
p=\frac{3 c-b^{2}}{3}, \quad q=\frac{2 b^{3}-9 b c+27 d}{27}, \quad r=2 \sqrt{-p / 3}, \quad w=\cos ^{-1}\left(\frac{3 q}{r p}\right), \quad \xi=-\frac{b}{3}
$$

Then, a condition for all roots being real is $p<0$ which is actually true in our case. These roots are given as

$$
\lambda_{k}=r \cos \left(\frac{w+(k-1) 2 \pi}{3}\right)+\xi, \quad k=1,2,3 .
$$

The corresponding three different eigenvectors are given as

$$
\mathbf{x}_{k}=\left[\begin{array}{c}
\frac{\lambda_{k}\left(\lambda_{k}-\alpha_{22}\right)+\alpha_{12}\left(\alpha_{33}-\lambda_{k}\right)+\alpha_{33}\left(\alpha_{22}-\lambda_{k}\right)-\alpha_{32}\left(\alpha_{13}+\alpha_{23}\right)}{\alpha_{32}\left(\alpha_{11}-\lambda_{k}+\alpha_{21}\right)-\alpha_{31}\left(\alpha_{22}-\lambda_{k}+\alpha_{12}\right)} \\
\frac{\alpha_{31}\left(\alpha_{13}+\alpha_{23}\right)+\left(\lambda_{k}-\alpha_{33}\right)\left(\alpha_{11}-\lambda_{k}+\alpha_{21}\right)}{\alpha_{32}\left(\alpha_{11}-\lambda_{k}+\alpha_{21}\right)-\alpha_{31}\left(\alpha_{22}-\lambda_{k}+\alpha_{12}\right)} \\
1
\end{array}\right], \quad k=1,2,3
$$

Based on the above eigenvectors, the transformation matrix $A$ can be expressed as

$$
A=\left(\begin{array}{lll}
\mathbf{x}_{1} & \mathbf{x}_{2} & \mathbf{x}_{3}
\end{array}\right)
$$

This transformation matrix linearly relates the actual and transformed dependent variables through the following relation

$$
\overline{\mathbf{c}}=A \overline{\mathbf{b}},
$$

were $\overline{\mathbf{c}}=\left(\bar{c}_{1}, \bar{c}_{2}, \bar{c}_{3}\right)^{T}$ and $\overline{\mathbf{b}}=\left(\bar{b}_{1}, \bar{b}_{2}, \bar{b}_{3}\right)^{T}$. After applying the above transformation on Eq. (21), we arrive at

$$
\frac{1}{P e_{k}} \frac{\partial^{2} \bar{b}_{k}}{\partial x^{2}}-\frac{\partial \bar{b}_{k}}{\partial x}-s \lambda_{k} \bar{b}_{k}=-\lambda_{k} b_{k, \text { init }}, \quad k=1,2,3
$$


Here

$$
\mathbf{b}_{\text {init }}=A^{-1} \mathbf{c}_{\text {init }}
$$

where $\mathbf{b}_{\text {init }}=\left(b_{1, \text { init }}, b_{2, \text { init }}, b_{3, \text { init }}\right)^{T}$ and $\mathbf{c}_{\text {init }}=\left(c_{1, \text { init }}, c_{2, \text { init }}, c_{3, \text { init }}\right)^{T}$.

The three decoupled steady-state advection-dispersion equations in Eq. (30) can be solved independently to get the following explicit solutions:

$$
\bar{b}_{k}(x, s)=A_{k} e^{m_{1}^{k} x}+B_{k} e^{m_{2}^{k} x}+\frac{b_{k, \text { init }}}{s}, \quad k=1,2,3
$$

where

$$
m_{1,2}^{k}=\frac{P e_{k} \pm \sqrt{P e_{k}^{2}+4 s P e_{k} \lambda_{k}}}{2}
$$

The boundary conditions in Eq. (18) are utilized to find the constants of integration $A_{k}$ and $B_{k}$ for $k=1,2,3$.

The Laplace transformations of BCs in Eq. (18) are given as

$$
\overline{c_{k}}(0, s)=\frac{c_{\mathrm{k}, \mathrm{inj}}}{s}\left(1-e^{-s \tau_{\mathrm{inj}}}\right), \quad \frac{\partial \overline{c_{k}}(\infty, s)}{\partial x}=0, \quad k=1,2,3 .
$$

The transformation of above equations into $\bar{b}$-domain gives

$$
\overline{\mathbf{b}}(0, s)=A^{-1} \overline{\mathbf{c}}(0, s), \quad \frac{\partial \bar{b}(\infty, s)}{\partial x}=0 .
$$

Using Eq. (35), the values of $A_{k}$ and $B_{k}$ in Eq. (32) take the following forms

$$
A_{k}=0, \quad B_{k}=\bar{b}_{k}(0, s)-\frac{b_{k, \text { init }}}{s}, \quad k=1,2,3
$$


After plugging these constants $A_{k}$ and $B_{k}$ in Eq. (32), we get

$$
\bar{b}_{k}(x, s)=\bar{b}_{k}(0, s) e^{m_{2}^{k} x}+\frac{b_{k, \text { init }}}{s}\left(1-e^{m_{2}^{k} x}\right), \quad k=1,2,3
$$

Finally, on using Eq. (37) in Eq. (29), we arrive at the following Laplace domain solutions

$$
\overline{\mathbf{c}}(x, s)=A \overline{\mathbf{b}}(x, s) .
$$

The analytical Laplace inversion is not possible in the current situation. However, it is easy to apply the numerical Laplace inversion by approximating the integral of the inverse Laplace transformation by Fourier series. ${ }^{42}$

\section{Moments analysis}

In this section, temporal moments are calculated by means of Laplace domain solutions. Moment analysis is an impressive tool to gain information which are very helpful to estimate kinetic parameters and to predict the shapes of elution profiles in a chromatographic process. In this paper, the analytical expressions of first two temporal moments are derived. The zeroth moment gives information about the peak area, while the first and second moments provide information about the retention time and variance (spreading) of the elution profile.

The following relations are used to derive moments from the Laplace domain solutions considering rectangular pulses injections. Let $\bar{w} \in\left\{\overline{c_{1}}, \overline{c_{2}}, \overline{c_{3}}\right\}$, then we have

$$
m_{w}^{0}=\frac{L}{u} \lim _{s \rightarrow 0}(\bar{w}(x=1, s)), \quad m_{w}^{n}=\left(-\frac{L}{u}\right)^{n} \lim _{s \rightarrow 0} \frac{d^{n}(\bar{w}(x=1, s))}{d s^{n}}, \quad n=1,2, \cdots
$$

For simplicity, it is assumed that $P e_{c}=P e_{T}$ and that column is regenerated initially 
having a reference temperature $T_{\text {ref }}$. Thus,

$$
c_{k, \text { init }}=0, \quad k=1,2,3 \text {. }
$$

Zeroth moments: The zeroth moments are given as

$$
m_{k}^{0}=c_{k, \text { inj }} t_{\text {inj }}, \quad k=1,2,3
$$

First moments: The expressions of first temporal moments are:

$$
\mathbf{m}^{1}=[\mu] \mathbf{m}^{0}
$$

Here, $\mathbf{m}^{0}=\left(m_{1}^{0}, m_{2}^{0}, m_{3}^{0}\right)^{T}, \mathbf{m}^{1}=\left(m_{1}^{1}, m_{2}^{1}, m_{3}^{1}\right)^{T}$ and the matrix $[\mu]$ is given as

$$
[\mu]=\left[\begin{array}{ccc}
\mu_{11} & \mu_{12} & \mu_{13} \\
\mu_{21} & \mu_{22} & \mu_{23} \\
\mu_{31} & \mu_{32} & \mu_{33}
\end{array}\right]=\frac{t_{\text {inj }}}{2}[I]+\frac{L}{u}\left[\begin{array}{ccc}
\alpha_{11} & \alpha_{12} & \alpha_{13} \\
\alpha_{21} & \alpha_{22} & \alpha_{23} \\
\alpha_{31} & \alpha_{32} & \alpha_{33}
\end{array}\right]
$$

where $[I]$ stands for $3 \times 3$ identity matrix and $\alpha_{k l}(k, l=1,2,3)$ are given by Eq. (16). Moreover, $\mu_{i j}$ demonstrates the mean-retention time of $i$ th component because of the input variation of $j$ th component.

Second central moments: The expressions of second temporal moments are:

$$
\mathbf{m}^{\prime 2}=[\sigma] \mathbf{m}^{0}=\left(\left[\mu^{2}\right]-\left[\mu^{1}\right]^{2}\right) \mathbf{m}^{0}
$$


where

$$
[\sigma]=\left[\begin{array}{ccc}
\sigma_{11} & \sigma_{12} & \sigma_{13} \\
\sigma_{21} & \sigma_{22} & \sigma_{23} \\
\sigma_{31} & \sigma_{32} & \sigma_{33}
\end{array}\right]=[I] \frac{t_{\text {inj }}^{2}}{12}+\frac{2 D_{z} L}{u^{3}}\left[\begin{array}{lll}
\alpha_{11} & \alpha_{12} & \alpha_{13} \\
\alpha_{21} & \alpha_{22} & \alpha_{23} \\
\alpha_{31} & \alpha_{32} & \alpha_{33}
\end{array}\right]^{2}
$$

Here, $\sigma_{i j}$ reveals the mean-variance of $i$ th component because of the input variation of $j$ th component.

\section{Numerical case studies}

This section presents some test problems for generating and analyzing concentration and temperature profiles obtained through the derived analytical solutions. A high resolution finite volume scheme (HR-FVS) is also used to numerically approximate the model equations for nonlinear isotherms given by Eqs. (1)-(5). ${ }^{43}$ The numerical and analytical results are compared with each other to gain confidence on the analytical results and to find the applicability ranges of the considered linearization. The proposed HR-FVS scheme has been thoroughly analyzed in our previous articles. ${ }^{24,25,43}$ The Matlab software was used for implementing both analytical and numerical methods in the computer. Principally, the two $\Delta H_{A, i}(i=1,2)$ can be distinct. However, from now on, we will take $\Delta H_{A, 1}=\Delta H_{A}=\Delta H_{A, 2}$. Table 1 summarizes the reference parameters of the case studies. The selected parameters lie in the ranges of parameters typically used in HPLC applications. 


\section{Influence of the enthalpy of adsorption $\Delta H_{A}$ for $T_{\mathrm{inj}}=T_{\mathrm{ref}}$ ("match- ing" injection)}

Figure 1 depicts the effects for an isothermal condition (considering $\Delta H_{A}=0$ ) and for three non-isothermal conditions (taking $\Delta H_{A}=-2,-10,-40 \mathrm{~kJ} / \mathrm{mol}$ ). Further, the inlet temperature of the sample has the defined reference value $\left(T_{\text {inj }}=T_{\text {ref }}\right)$. Figure 1a indicates that when the enthalpy of adsorption is zero, no change in the temperature profile occurs. On the other hand, as visible in Figures 1b, 1c \& 1d; the enthalpy of adsorption significantly effects the temperature profile $c_{3}=T-T_{\text {ref }}$, without making pronounced changes in the concentration profiles $c_{1}$ and $c_{2}$. It can be seen that moderate values of the adsorption enthalpy $\left(\Delta H_{A}-2 \mathrm{~kJ} / \mathrm{mol}\right.$ and $\left.\Delta H_{A}-10 \mathrm{~kJ} / \mathrm{mol}\right)$ produce considerable rise in the temperature profile $(>0.08 K$ and $>0.4 K)$. One can further observe in Figure 1d that rise in the temperature profile is much higher $(>1.7 \mathrm{~K})$ for the larger value of the enthalpy of adsorption (e.g. $\Delta H_{A}=-40 \mathrm{~kJ} / \mathrm{mol}$ ). Afterwards, desorption of each component occurs which is connected to the reduction of temperature. At last, after passing the profiles, the temperature profile comes back to its reference value.

Figures 2 and 3 depict the comparison of analytical solutions for linearized isotherms (c.f. Eq. (38)) and numerical solutions of HR-FVS for nonlinear isotherms (c.f. Eq. (3)) at different values of $\Delta H_{A}$ which is assumed identical for both components. It can be noticed that analytical and numerical solutions start moving away from each other when the magnitude of enthalpy of adsorption, $\left|\Delta H_{A}\right|$, exceed $10 \mathrm{~kJ} / \mathrm{mol}$. Thus, large values of the enthalpy of adsorption are mainly responsible for prominent temperature variations. All the figures clearly support our linear assumptions for moderate values of the enthalpy of adsorption. For the enthalpy of adsorption $-40 \mathrm{~kJ} / \mathrm{mol}$, one can see large temperature deviations (Figure 3). An overestimation can be seen in our analytical results as compared to the numerical results in those parts of the solution which show deviation from the 
isothermal behavior.

\section{Influence of ratio $\rho^{S} c_{p}^{S} / \rho^{L} c_{p}^{L}$}

Figure 4 depicts the influence of ratio $\rho^{S} c_{p}^{S} / \rho^{L} c_{p}^{L}$ on the shapes of concentration and temperature profiles. In Figure 4a, for $\rho^{S} c_{p}^{S} / \rho^{L} c_{p}^{L}=1$, the adsorption peak of temperature profile is moving slightly faster as compared to the concentration profiles. In Figure 4b, for the ratio $\rho^{S} c_{p}^{S} / \rho^{L} c_{p}^{L}<1$, the concentration profiles are moving much slower than the temperature profile. The decoupled adsorption peak of the temperature profile is moving faster than the adsorption peak of concentration, while the desorption peaks of temperature are coupled with concentration profiles, i.e. they are moving at the same speed. In Figure 4c, for the ratio $\rho^{S} c_{p}^{S} / \rho^{L} c_{p}^{L}>1$, the speed of temperature and concentration profiles are almost the same. Both temperature and concentration profiles leave the column at almost the same time.

\section{Affect of nonlinearity coefficients $b_{i}$}

Figure 5 gives a comparison of analytical solutions for linearized isotherms (c.f. Eq. (38)) together with numerical solutions of HR-FVS for nonlinear isotherms (c.f. Eq. (3)) for two distinct values of $b_{i}(i=1,2)$. It can be seen in Figures $5 \mathrm{a}, 5 \mathrm{~b}$ that analytical and numerical solutions agree with each other for small values of $b_{i}$, i.e. for $b_{1}=b_{2}=0.01$. However, as $b_{1}$ and $b_{2}$ increase to 0.05 both solutions start deviating form each other as

shown in Figures 5c, 5d. This proves that our current analytical solutions are only valid for small values of nonlinearity coefficients. 


\section{Affect of injection temperature $\left(T_{\mathrm{inj}}\right)$}

The case study in Figure 6 demonstrates that how a variation in the temperature of the injected sample effects the profile of temperature during its propagation through the column. One can clearly observe in Figure 6a that the faster peak of adsorption in the temperature profile rises further with the hot injection $\left(T_{\mathrm{inj}}>T_{\text {ref }}\right)$, whereas a mirror effect can be seen on the same peak in Figure $6 \mathrm{~b}$ for the cold injection $\left(T_{\mathrm{inj}}<T_{\mathrm{ref}}\right)$.

It is important to mention that both types of injection have no visible influence on the concentration profiles due to small temperature amplitudes generated by low values of the enthalpy of adsorption.

\section{Affects of Henry's constants $\left(a_{i, \mathrm{ref}}\right)$}

Figure 7 shows the plots of concentrations and temperature profiles for distinct values of the reference Henry's constants $a_{i, \text { ref }}(i=1,2)$. As the values of Henry's constants increases, the retention times of concentration and temperature profiles attain larger values and peaks become wider. The amplitudes of concentration profiles decreases and peak of the temperature profile increases for larger values of $a_{i, \text { ref }}$.

\section{Affects of $u$ on temporal moments}

Figure 8 shows the effects of $u$ on the first and second moments expressing retention times

and variances of the elution profiles. In these plots, we have taken $\rho^{S} c_{p}^{S}=40 \mathrm{~kJ} / \mathrm{lK}$ and $\rho^{L} c_{p}^{L}=4 k J / l K$. It can be observed in Figure 8a that $u$ has no effect on $\mu_{12}$ and $\mu_{21}$ which means that the diffusion of one component is not influenced by other one, whereas $\mu_{11}$ and $\mu_{22}$ show a decrease in retention time with increasing velocity $u$. Further Figure 8b shows that $u$ does not influences $\mu_{13}$ and $\mu_{23}$, however, $\mu_{31}$ and $\mu_{32}$ increases and $\mu_{33}$ decreases on increasing the velocity $u$. On the other hand, Figure 8c shows that variances $\sigma_{11}$ and $\sigma_{22}$ 
are decreasing with increasing $u$, whereas $\sigma_{12}$ and $\sigma_{21}$ are not effected or remain constant throughout. Figure 8d shows that variances $\sigma_{13}$ and $\sigma_{23}$ are not effected or have constant behavior, $\sigma_{33}$ decreases with increasing $u$, whereas $\sigma_{31}$ and $\sigma_{32}$ increases with increasing $u$. The trends observed in these moments plots agree well with those found in the elution profiles shown in Figures 8a-d.

\section{Conclusion}

A two-component linearized non-isothermal equilibrium dispersive model (EDM) has been analyzed analytically and numerically. The Laplace transformation, the eigen-decomposition technique, along with the elementary solution technique of ODEs have been applied simultaneously to find the analytical solutions of the model equations. These analytical solutions are important to analyze and understand the dynamics of concentration and thermal fronts in chromatographic columns considering sufficiently small nonlinearity and deviations from isothermal conditions. In order to get a detailed analysis, analytical expressions of the first and second temporal moments were derived by utilizing the moment generating property of the solutions in the Laplace domain. The derived analytical expressions of moments are very helpful to understand the dynamics and shapes of outlet concentration and temperature profiles, as well as for estimating kinetic and thermodynamic parameters by equating the experimental and theoretical moments. Although not exercised practically, application would demand a careful recording of a small variations in the temperature. Some interesting cases and differences in the retention times and variances of two-component adsorption system have been discussed and shown graphically to understand and analyze the behavior of the multi-component non-isothermal system. To validate their applicability ranges, the analytical solutions for linearized model are compared with the numerical results of a nonlinear model. It was found that these analytical solutions are well applicable for the 
small values of the enthalpy of adsorption and nonlinearity coefficients.

\section{Abbreviations}

BCs, boundary conditions; EDM, equilibrium dispersive model; HETP, height equivalent to the theoretical plate; HR-FVS, high resolution finite volume scheme; ODEs, ordinary differential equations; Pe, peclet number.

\section{Symbols}

$a_{i}^{\text {ref }}$, Henry constant; $b_{j}^{\text {ref }}$, coefficient of nonlinearity; $c_{i}$, concentrations of the $i$ th component solute in the mobile phase; $c_{p}$, heat capacity; $D_{z}$, axial dispersion coefficient; $\Delta H_{A}$, enthalpy of adsorption; $L$, length of the column; $P e_{c}$, Peclet number for the concentration; $P e_{T}$, Peclet number for the temperature; $q_{i}^{*}$, concentrations of the $i$ th component solute in the adsorbed phase; $R_{g}$, general gas constant; $t$, time coordinate; $T$, bulk temperature; $T_{\text {init }}$, initial temperature; $T_{\text {inj }}$, inlet temperature; $T_{\text {ref }}$, reference temperature; $u$, interstitial velocity; $x$, axial coordinate; $\epsilon$, total porosity; $\rho$, density per unit volume; $\lambda_{z}$, axial heat conductivity; $\delta_{k l}$, Kronecker delta function; $\rho^{L} c_{p}^{L}$, heat capacity of liquid times density; $\rho^{S} c_{p}^{S}$, heat capacity of solid times density.

\section{References}

(1) Glueckauf, E. Theory of Chromatography. VII. The General Theory of Two Solutes following Non-linear Isotherms. Discuss. Faraday Soc. 1949, 7, 12.

(2) Rhee, H.K.; Aris, R.; Amundson, N.R. On the Theory of Multicomponent Chromatography. Philos. Trans. Royal Soc. A 1970, 267, 419. 
(3) Ruthven, D.M. Principles of Adsorption and Adsorption Processes; John Wiley and Sons: New York, 1984.

(4) Yang, R.T. Gas Separation by Adsorption Processes; Butterworth-Heinemann: Boston, 1987.

(5) Deans, H.A.; Horn, F.J.M.; Klauser, G. Perturbation Chromatography in Chemically Reactive Systems. AIChE J. 1970, 16, 426.

(6) Guiochon, G. Preparative Liquid Chromatography. J. Chromatogr. A 2002, 965, 129.

(7) Guiochon, G.; Lin, B. Modeling for Preparative Chromatography; Academic Press: London, 2003.

(8) Guiochon, G.; Felinger, A.; Shirazi, D.G., Katti, A.M. Fundamentals of Preparative and Nonlinear Chromatography, 2nd ed.; Elsevier Academic Press: New York, 2006.

(9) Sainio, T.; Zhang, L.; Seidel-Morgenstern, A. Adiabatic Operation of Chromatographic Fixed-bed Reactors. Chem. Eng. J. 2011, 168, 861.

(10) Sainio, T.; Kaspereit, M.; Kienle, A.; Seidel-Morgenstern, A. Thermal Effects in Reactive Liquid Chromatography. Chem. Eng. Sci. 2007, 62, 5674.

(11) Vu, T.D.; Seidel-Morgenstern, A. Quantifying Temperature and Flow Rate Effects on the Performance of a Fixed-bed Chromatographic Reactor. J. Chromatogr. A 2011, $1218,8097$.

(12) Gritti, F.; Gilar, M.; Jarrell, J.A. Quasi-adiabatic Vacuum-based Column Housing for very High-pressure Liquid Chromatography. J. Chromatogr. A 2016, 1456, 226.

(13) Carslaw, H.S.; Jaeger, J.C. Conduction of Heat in Solids, 2nd ed.; Clarendon Press: Oxford, 1959. 
(14) Eigenberger, G.; Kolios, G.; Nieken, U. Efficient Reheating of a Reverse-flow Reformer-An Experimental Study. Chem. Eng. Sci. 2007, 62, 4825.

(15) Glöckler, B.; Dieter, H.; Eigenberger, G.; Nieken, U. Efficient Reheating of a Reverseflow Reformer-An Experimental Study. Chem. Eng. Sci. 2007, 62, 5638.

(16) Kruglov, A. V. Methanol Synthesis in a Simulated Countercurrent Moving-bed Adsorptive Catalytic Reactor. Chem. Eng. Sci. 1994, 49, 4699.

(17) Yongsunthon, I.; Alpay, E. Design of Periodic Adsorptive Reactors for the Optimal Integration of Reaction, Separation and Heat Exchange. Chem. Eng. Sci. 1999, 54, 2647.

(18) Brandt, A.; Mann, G.; Arlt, W. Temperature Gradients in Preparative Highperformance Liquid Chromatography Columns. J. Chromatogr. A 1997, 769, 109.

(19) Cerro, R.L.; Smith, W. Effects of Heat Release and Nonlinear Equilibrium on Transient Adsorption. Ind. Eng. Chem. Fundam. 1969, 8, 796.

(20) Haynes, Jr. H. W. An Analysis of Sorption Heat Effects in the Pulse Gas Chromatography Diffusion Experiment. AIChE J. 1986, 32, 1750.

(21) Sainio, T. Ion-exchange Resins as Stationary Phase in Reactive Chromatography. Ph.D. Dissertation, Lappeenranta University of Technology, Lappeenranta, Finland, 2005.

(22) McCalley, D.V. Effect of Temperature and Flow-rate on Analysis of Basic Compounds in High-performance Liquid Chromatography Using a Reversed-phase Column. J. Chromatogr. A 2000, 902, 311.

(23) Zhong, G. M.; Meunier, F.; Guiochon, G. Interference and Heat Effects: Moment Analysis for Two-component Chromatography. J. Chromatogr. A 1994, 658, 355. 
(24) Javeed, S.; Qamar, S.; Seidel-Morgenstern, A.; Warnecke, G. Parametric Study of Thermal Effects in Reactive Liquid Chromatography. Chem. Eng. J. 2012, 191, 426.

(25) Qamar, S.; Sattar, F.A.; Batool, I.; Seidel-Morgenstern, A. Theoretical Analysis of the Influence of Forced and Inherent Temperature Fluctuations in an Adiabatic Chromatographic Column. Chem. Eng. Sci. 2017, 161, 249.

(26) Martin, M.; Guiochon, G. Effects of High Pressure in Liquid Chromatography. J. Chromatogr. A 2005, 1090, 16.

(27) Van Genuchten, M.Th. Analytical Solutions for Chemical Transport with Simultaneous Adsorption, Zero-order Production and First-order Decay. J. Hydrol 1981, 49, 213.

(28) Javeed, S.; Qamar, S.; Ashraf, W.; Warnecke, G.; Seidel-Morgenstern, A. Analysis and Numerical Investigation of Two Dynamic Models for Liquid Chromatography. Chem. Eng. Sci. 2013, 90, 17.

(29) Qamar, S.; Seidel-Morgenstern, A. Extending the Potential of Moment Analysis in Chromatography. TrAC, Trends Anal. Chem. 2016, 81, 87.

(30) Rice, R.G.; Do, D.D. Applied Mathematics and Modeling for Chemical Engineers; John Wiley and Sons: New York, 1995.

(31) Widder, D. V. Laplace Transform (PMS-6); Princeton University Press: Princeton, New Jersey, 2015.

(32) Kubin, M. Beitrag zur Theorie der Chromatographie II. Einfluss der Diffusion Ausserhalb und der Adsorption Innerhalb des Sorbens-Korns. Collect. Czech. Chem. Commun. 1965, 30, 2900. 
(33) Kubin, M. Beitrag zur Theorie der Chromatographie. Collect. Czech. Chem. Commun. 1965, 30, 1104 .

(34) Kucera, E. Contribution to the Theory of Chromatography: Linear Non-equilibrium Elution Chromatography. J. Chromatogr. A 1965, 19, 237.

(35) Lenhoff, A.M. Significance and Estimation of Chromatographic Parameters. J. Chromatogr. A 1987, 384, 285.

(36) Miyabe, K.; Guiochon, G. Influence of the Modification Conditions of Alkyl Bonded Ligands on the Characteristics of Reversed-phase Liquid Chromatography. J. Chromatogr. A 2000, 903, 1.

(37) Miyabe, K. Moment Analysis of Chromatographic Behavior in Reversed-phase Liquid Chromatography. J. Sep. Sci. 2009, 32, 757.

(38) Schneider, P.; Smith, J.M. Adsorption Rate Constants from Chromatography. AIChE J. 1968, 14, 762 .

(39) Miyabe, K.; Guiochon, G. Measurement of the Parameters of the Mass Transfer Kinetics in High Performance Liquid Chromatography. J. Sep. Sci. 2003, 26, 155.

(40) Suzuki, M. Notes on Determining the Moments of the Impulse Response of the Basic Transformed Equations. J. Chem. Eng. Jpn. 1974, 6, 540.

(41) Wolff, H.-J.; Radeke, K.-H; Gelbin, D. Heat and Mass Transfer in Packed Beds-IV: Use of Weighted Moments to Determine Axial Dispersion Coefficients. Chem. Eng. Sci. 1979, 34, 101.

(42) Durbin, F. Numerical Inversion of Laplace Transforms: An Efficient Improvement to Dubner and Abate's Method. Comput. J. 1974, 17, 371. 
(43) Javeed, S.; Qamar, S.; Seidel-Morgenstern, A.; Warnecke, G. Efficient and Accurate Numerical Simulation of Nonlinear Chromatographic Processes. J. Comput. \&3 Chem.

Eng. 2011, 35, 2294.

Table 1. Reference parameters used in the case studies.

\begin{tabular}{|l|l|}
\hline Parameters & Values \\
\hline \hline Length of column & $L=10 \mathrm{~cm}$ \\
Henry's constants & $a_{1, \text { ref }}=1.4, a_{2, \text { ref }}=2.0$ \\
Nonlinearity coefficients & $b_{1, \text { ref }}=b_{2, \text { ref }}=0.01$ \\
Interstitial velocity & $u=1.0 \mathrm{~cm} / \mathrm{min}$ \\
Porosity & $\epsilon=0.4$ \\
Heat capacity of liquid times density & $c_{p}^{L} \rho^{L}=4 \mathrm{~kJ} / \mathrm{lK}$ \\
Heat capacity of solid times density & $c_{p}^{S} \rho^{S}=40 \mathrm{~kJ} / \mathrm{lK}$ \\
Adsorption Enthalpy & $\Delta H_{A}=-2 \mathrm{~kJ} / \mathrm{mol}^{S}$ \\
Dispersion coefficient & $D_{z}=0.02 \mathrm{~cm}^{2} / \mathrm{min}^{-1}$ \\
Heat conductivity coefficient & $\lambda_{z}=0.08 \mathrm{~kJ} \mathrm{~cm} \mathrm{~min}^{-1}$ \\
Initial concentrations & $c_{i, \text { init }}=0 \mathrm{~mol} / l(i=1,2)$ \\
Inlet concentrations & $c_{i, \text { inj }}=1 \mathrm{~mol} / l(i=1,2)$ \\
Initial temperature & $T_{\text {init }}=300 \mathrm{~K}$ \\
Inlet temperature & $T_{\text {inj }}=300 \mathrm{~K}$ \\
Reference temperature & $T_{\text {ref }}=300 \mathrm{~K}$ \\
Injection time & $t_{\text {inj }}=5 \mathrm{~min}$ \\
\hline
\end{tabular}



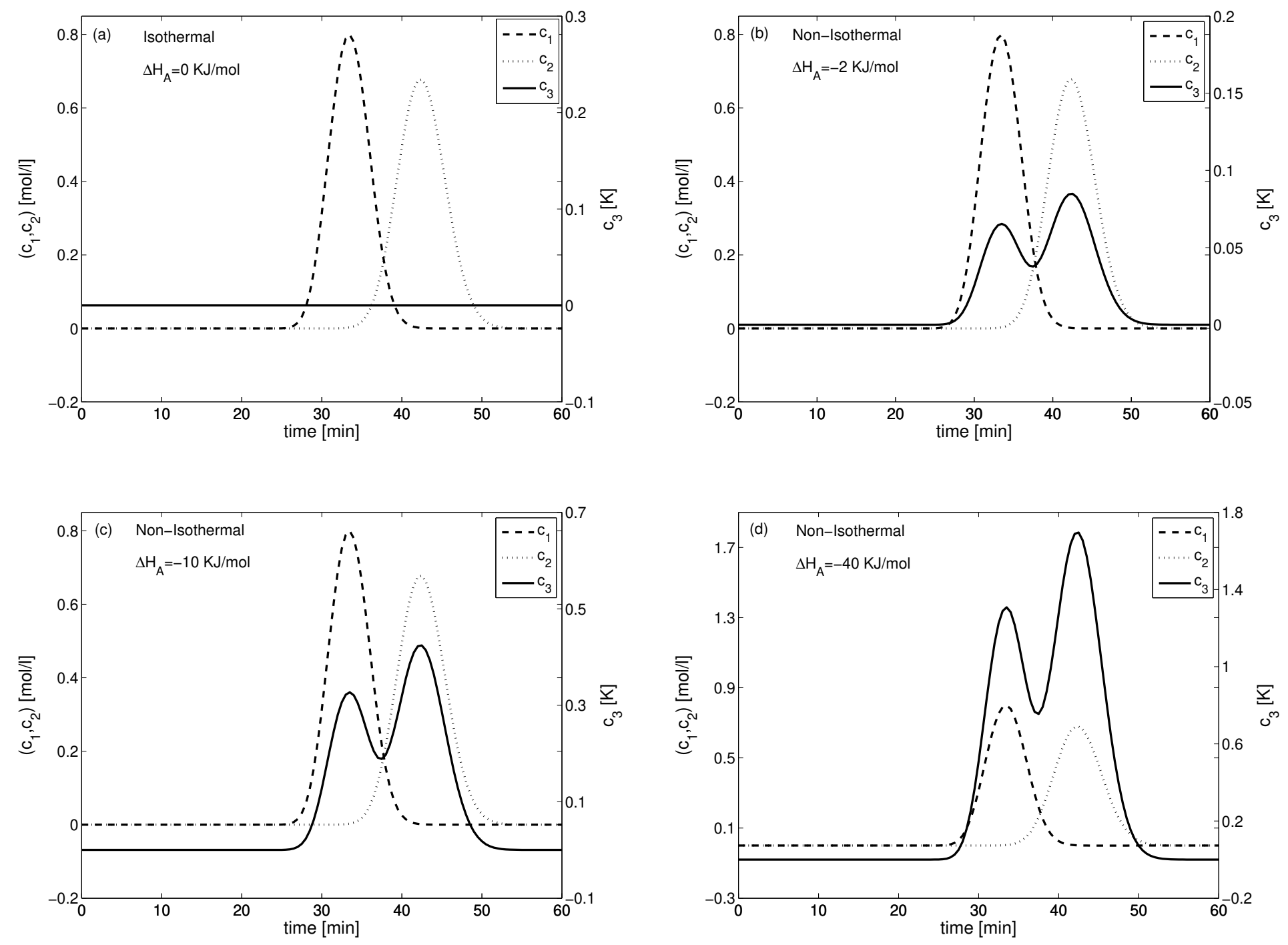

Figure 1. Effect of $\Delta H_{A}$ on concentration profiles $\left(c_{1} \& c_{2}\right)$, and on temperature profile $\left(c_{3}\right)$ for $T_{\text {inj }}=T_{\text {ref }}=300 K, \rho^{S} c_{p}^{S}=40 k J / l k, \rho^{L} c_{p}^{L}=4 k J / l k$. All other parameters are given in Table 1. 

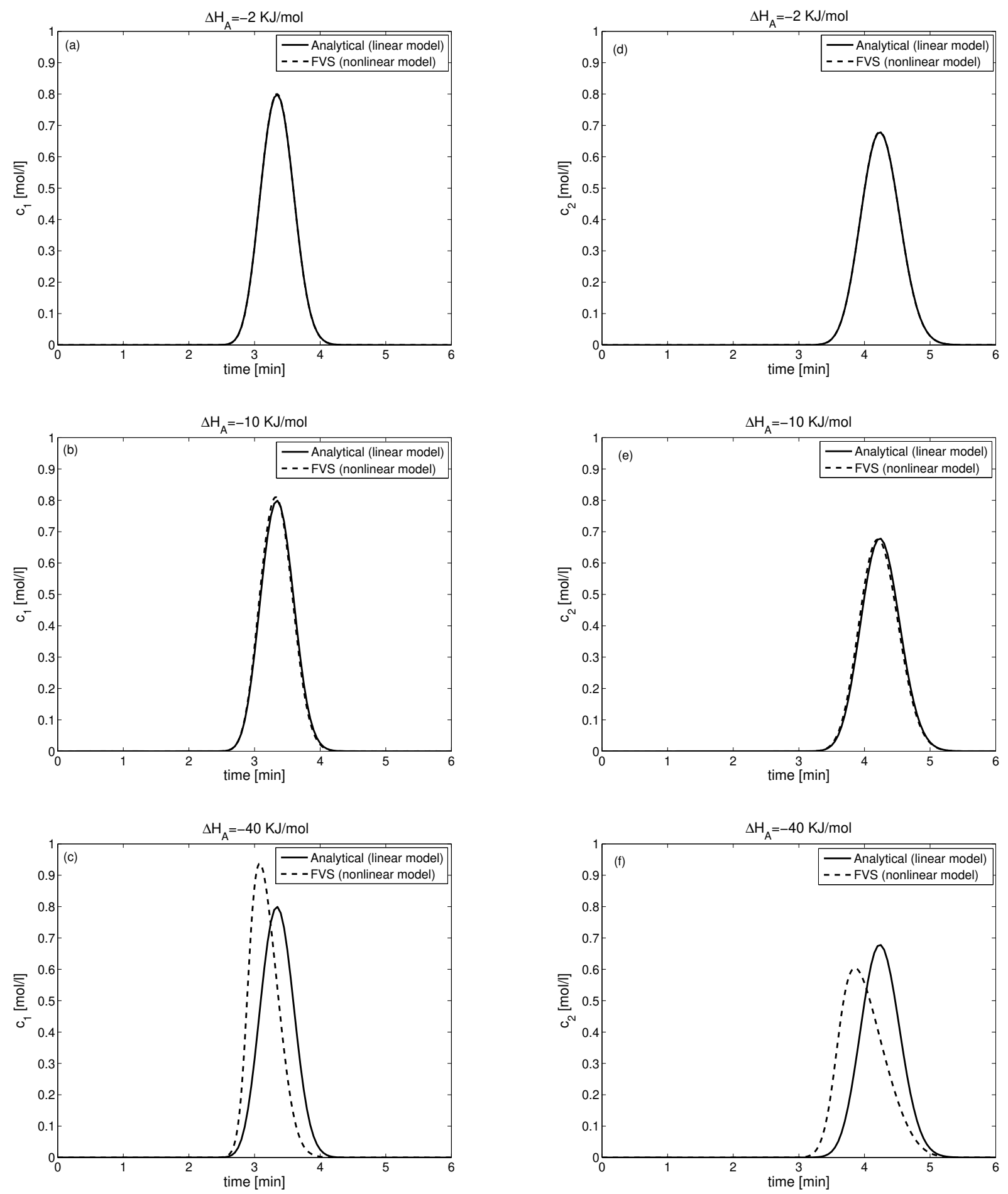

Figure 2. A comparison of analytically and numerically calculated concentration profiles $\left(c_{1} \& c_{2}\right)$ at different values of the enthalpy of adsorption $\Delta H_{A}$. Numerical solutions are based on nonlinear isotherm (c.f. Eq. (3)) and analytical solutions are based on linearized isotherm (c.f. Eq. (9)). Here, $T_{\text {inj }}=T_{\text {init }}=T_{\text {ref }}, \rho^{S} c_{p}^{S}=40 k J / l k, \rho^{L} c_{p}^{L}=4 k J / l k$, and all other parameters are given in Table 1. 

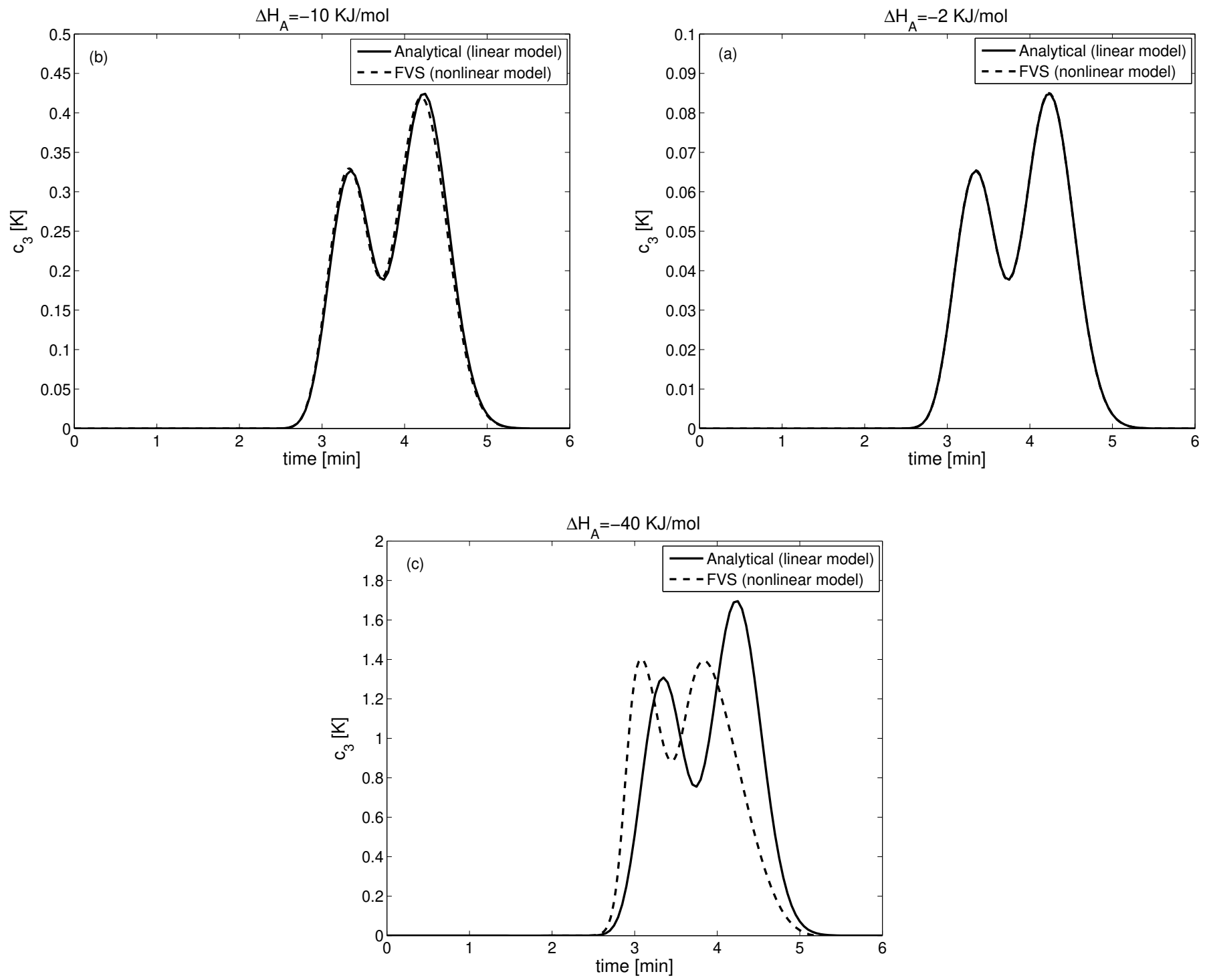

Figure 3. A comparison of analytically and numerically calculated temperature profiles $\left(c_{3}\right)$ at different values of the enthalpy of adsorption $\Delta H_{A}$. Numerical solutions are based on nonlinear isotherm (c.f. Eq. (3)) and analytical solutions are based on linearized isotherm (c.f. Eq. (9)). Here, $T_{\text {inj }}=T_{\text {init }}=T_{\text {ref }}, \rho^{S} c_{p}^{S}=40 k J / l k, \rho^{L} c_{p}^{L}=4 k J / l k$, and all other parameters are given in Table 1. 

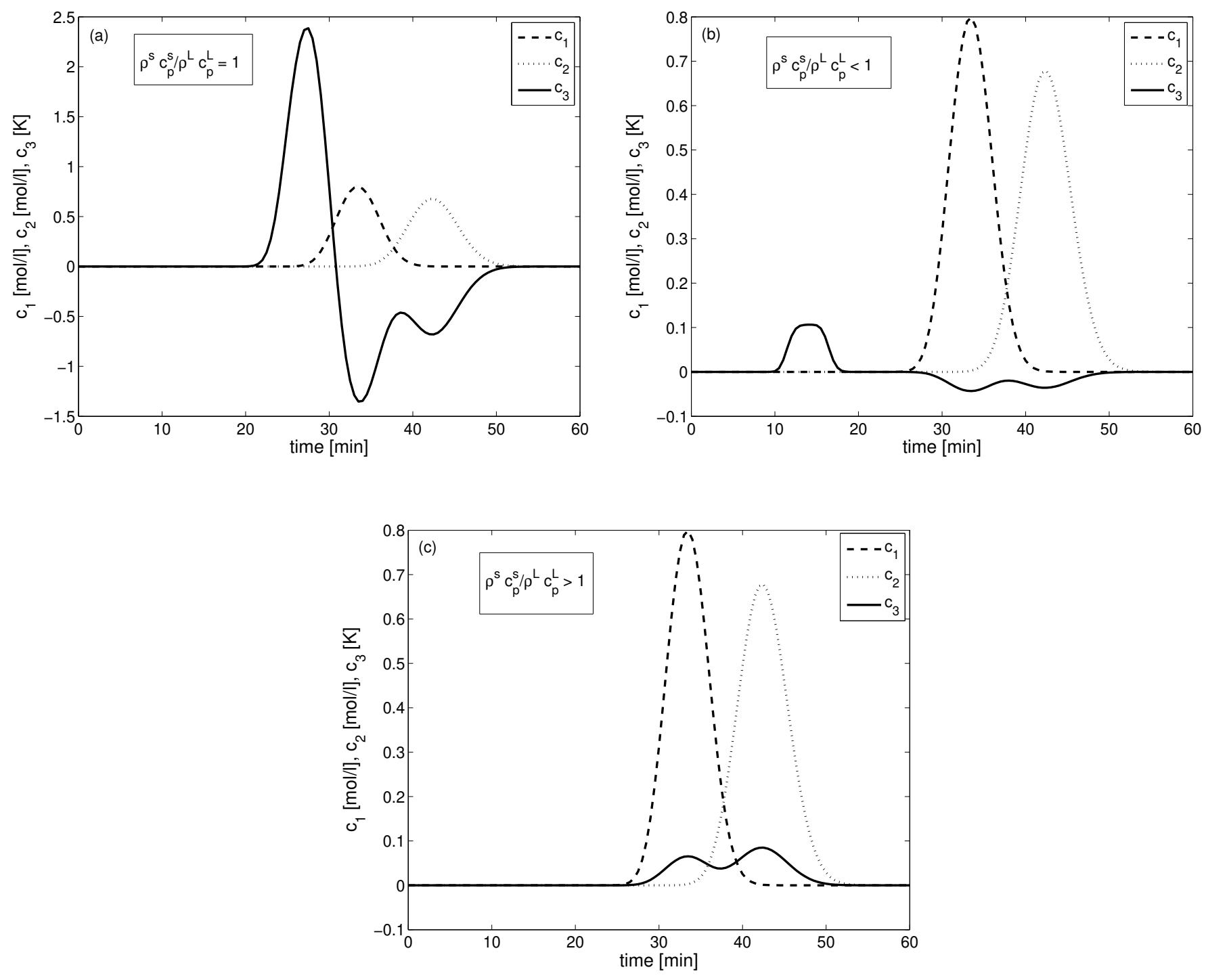

Figure 4. Effect of $\rho^{S} c_{p}^{S} / \rho^{L} c_{p}^{L}$ on concentration profiles $\left(c_{1} \& c_{2}\right)$, and temperature profile $\left(c_{3}\right)$. Plot (a) $\rho^{S} c_{p}^{S}=4 k J / l k$ and $\rho^{L} c_{p}^{L}=4 k J / l k$, plot (b) $\rho^{S} c_{p}^{S}=4 k J / l k$ and $\rho^{L} c_{p}^{L}=$ $40 \mathrm{~kJ} / \mathrm{lk}$, plot (c) $\rho^{S} c_{p}^{S}=40 \mathrm{~kJ} / \mathrm{lk}$ and $\rho^{L} c_{p}^{L}=4 \mathrm{~kJ} / \mathrm{lk}$. Here, $T_{\mathrm{inj}}=T_{\text {ref }}=300 \mathrm{~K}$ and other parameters are given in Table 1. 

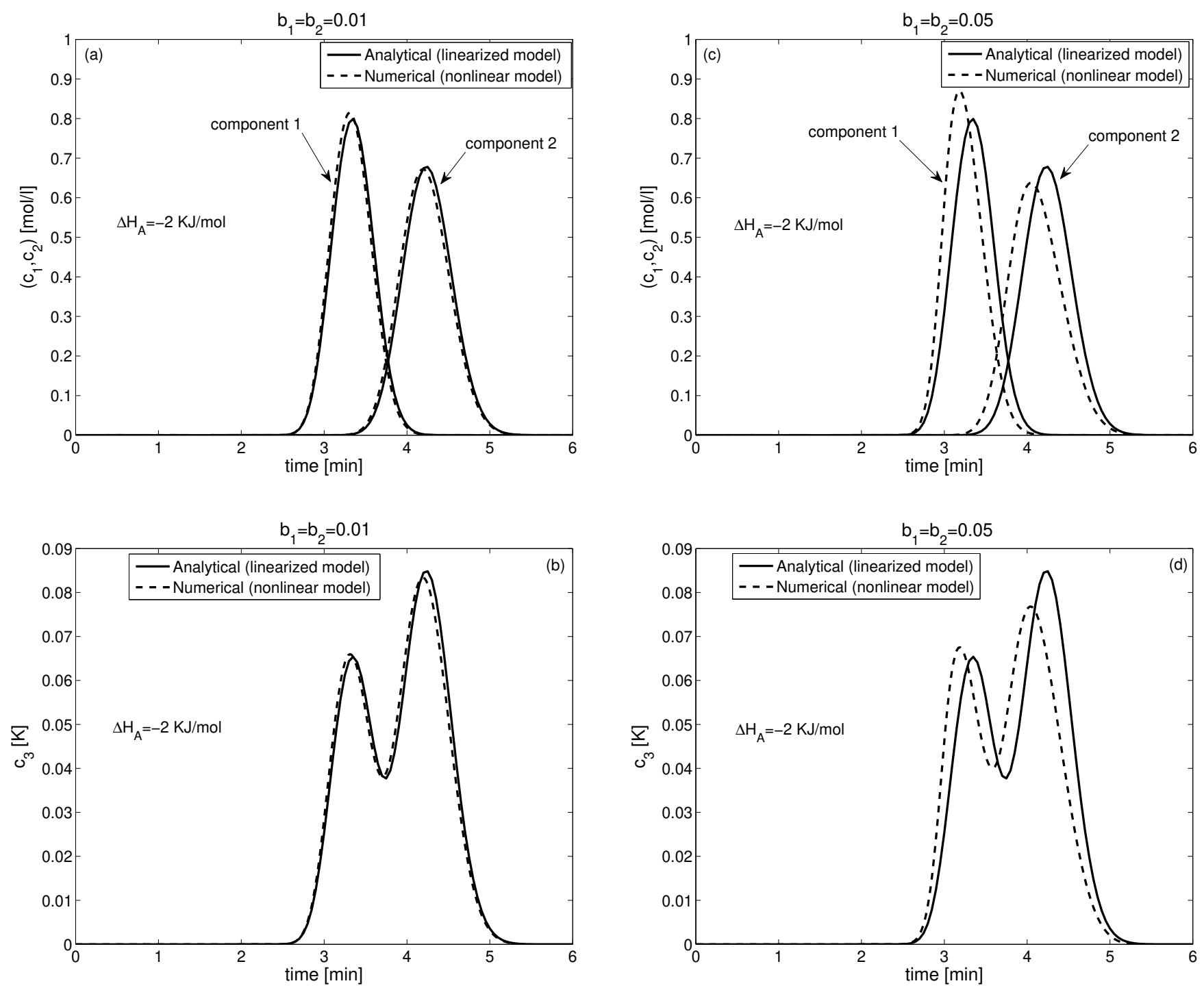

Figure 5. A comparison of analytical solutions for linear isotherm (c.f. Eq. (9)) and numerical solutions for nonlinear isotherm (c.f. Eq. (3)) for two different values of $b_{i}(=1,2)$. Plots (a) and (c) show the effect of non-linearity $b_{i}$ on the concentration profiles $\left(c_{1} \& c_{2}\right)$ and plots $(\mathrm{b})$ and $(\mathrm{d})$ show the effect of non-linearity $b_{i}$ on temperature profile $\left(c_{3}\right)$. In addition, $T_{\text {inj }}=T_{\text {init }}=T_{\text {ref }}, \rho^{S} c_{p}^{S}=40 k J / l k, \rho^{L} c_{p}^{L}=4 k J / l k$ and all other parameters are given in Table 1. 

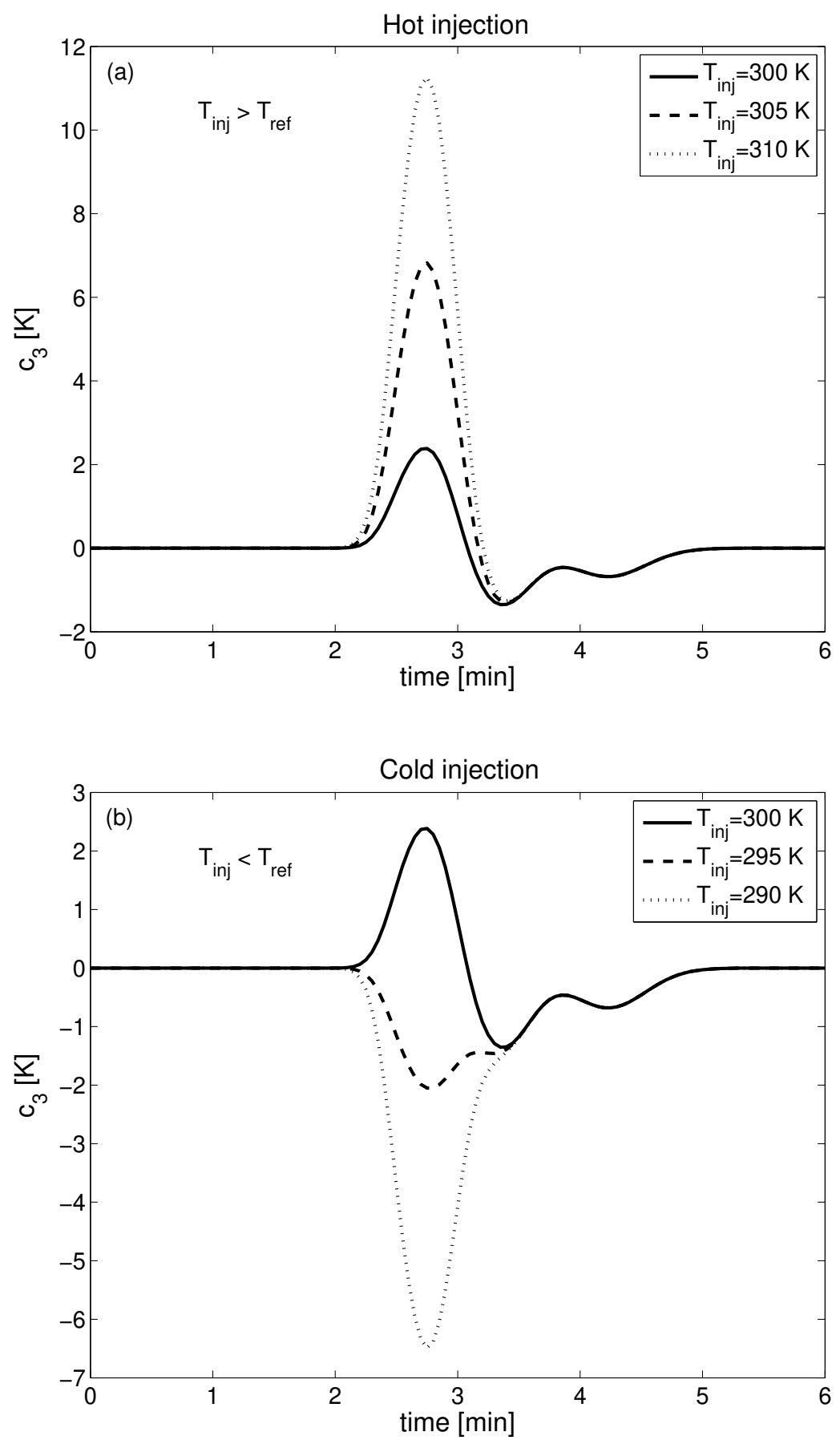

Figure 6. Effect of $T_{\text {inj }} \neq T_{\text {ref }}$ for $\rho^{S} c_{p}^{S}=4 k J / l k$ and $\rho^{L} c_{p}^{L}=4 k J / l k$ are shown in plots (a) and (b). The effect for $\rho^{S} c_{p}^{S}=40 k J / l k$ and $\rho^{L} c_{p}^{L}=4 k J / l k$ was very negligible and is, therefore, omitted. Other parameters used in the simulation are given in Table 1. 

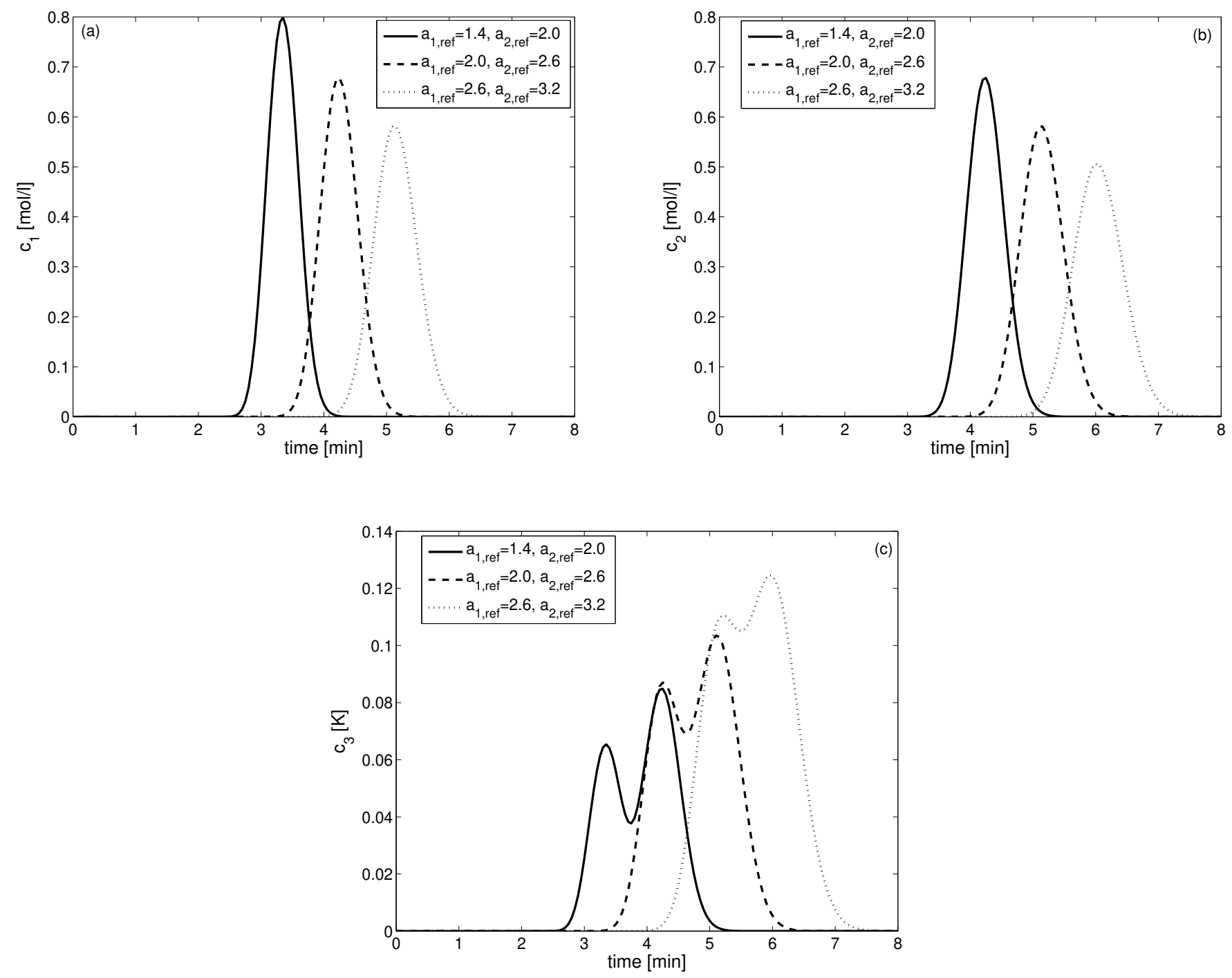

Figure 7. The effects of Henry's constants $a_{i, \text { ref }}$ for three different sets of values on concentration profiles (plots (a) \& (b)) and temperature profile (plot (c)). Here, $\rho^{S} c_{p}^{S}=40 \mathrm{~kJ} / \mathrm{lk}$, $\rho^{L} c_{p}^{L}=4 k J / l k$ and other parameters are given in Table 1. 



Figure 8. Effect of velocity $u$ on the concentrations and temperature moments. Here, $T_{\mathrm{inj}}=T_{\mathrm{init}}=T_{\mathrm{ref}}, \rho^{S} c_{p}^{S}=40 \mathrm{~kJ} / l k, \rho^{L} c_{p}^{L}=4 k J / l k$ and other parameters are given in Table 1. 


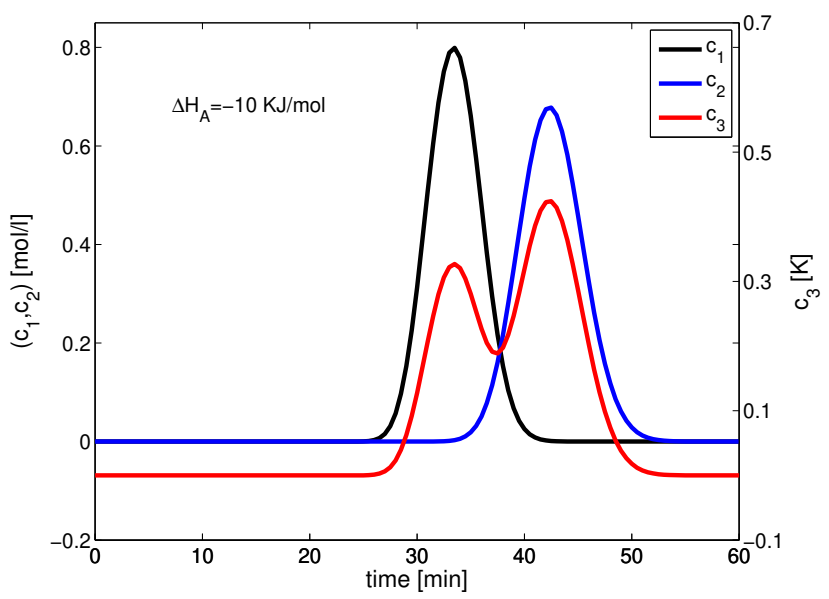

For Table of Contents only. 